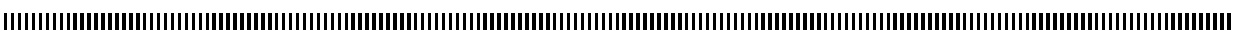

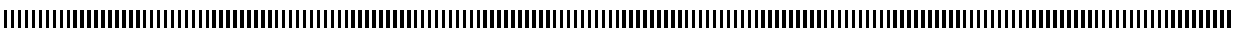

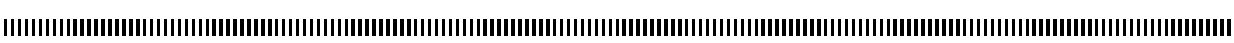

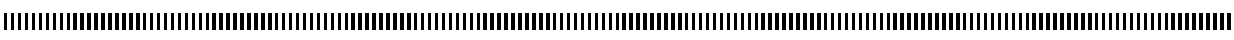

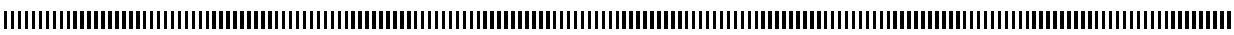

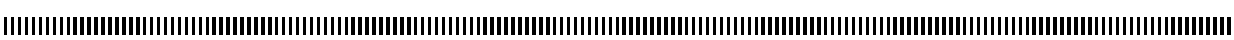

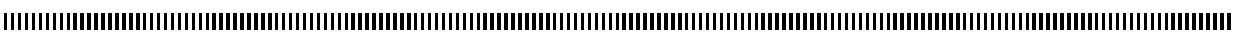

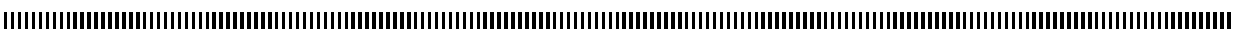

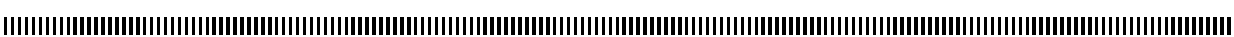

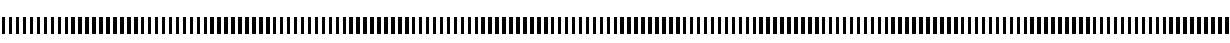

\title{
Résolution d'un Problème de Cauchy en EEG
}

\author{
Abdellatif EL-BADIA, Maha FARAH, Tuong HA-DUONG, Vincent PAVAN* \\ * Université de Technologie de Compiègne - Laboratoire de Mathématiques Appliquées \\ B.P. 20529 - 60205 Compiegne Cedex - FRANCE \\ Email : mfarah@dma.utc.fr

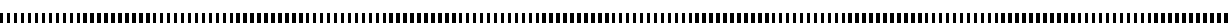

RÉSUMÉ. Dans cet article, nous traitons un problème de Cauchy dans le cadre de la localisation des sources épileptiques en Electro-Encéphalo-Graphie (EEG). Plus particulièrement, il s'agit du problème de construction des données de Cauchy sur la surface du cerveau à partir des données du potentiel mesuré par l'EEG à la surface de la tête. Notre résolution est basée sur un algorithme itératif alternatif initialement proposé par Kozlov, Maz'ya et Fomin [16]. Nous présentons dans ce papier l'étude numérique de cette méthode que nous avons implémentée dans $R^{3}$. Nous donnons également des applications et des résultats numériques.

ABSTRACT. This paper talks about the resolution of the Cauchy problem thats appears in the localization of epileptic sources on Electro-Encephalo-Graphy (EEG). We treat specially the problem of estimating Cauchy data over the layer of the brain, knowing only the ones on the scalp measured by EEG. As a method of resolution, we choose an alternating iteratif algorithm first proposed by Kozlov, Maz'ya and Fomin [16]. In this paper, we study numerically this method in $R^{3}$. We give also some numerical examples.

MOTS-CLÉS : Problème de Cauchy, Equations intégrales, Méthode alternative itérative, ElectroEncéphalo-Graphie (EEG).

KEYWORDS : Cauchy Problem, Integral Equations, Alternating Iterative Method, Electro-EncephaloGraphy (EEG).

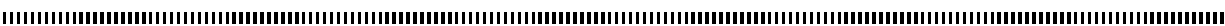

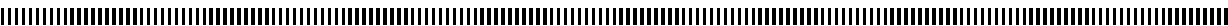
|

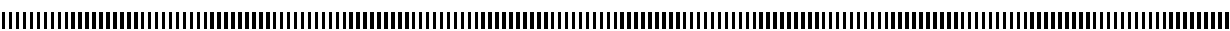
| | |

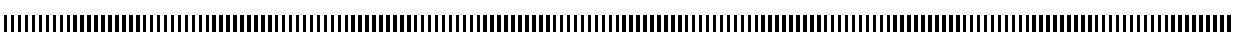
| 


\section{TamTam'05}

\section{Introduction}

Le cadre de ce papier se situe dans le thème des problèmes inverses de sources en ElectroEncéphalo-Graphie (EEG). Il s'agit de déterminer la localisation de foyers épileptogènes qui sont à l'origine de signaux EEG mesurés au moyen d'électrodes placées sur le scalp. C'est une méthode non invasive dont la résolution temporelle est l'ordre de la milliseconde [12].

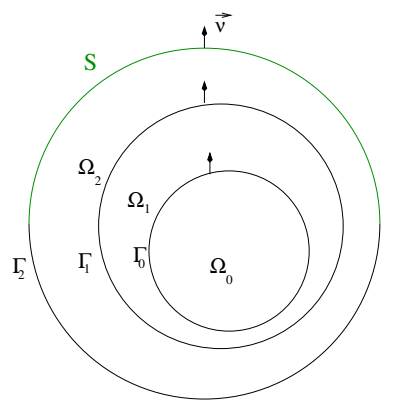

Figure 1.

Le problème direct en EEG consiste à calculer les potentiels recueillis sur la peau qu'engendrent une configuration de sources connues. Cela nécessite une bonne connaissance de la géométrie de la tête ainsi que les propriétés de conduction dans les tissus cérébraux [9].

Le problème inverse qui nous concerne ici, consiste à estimer la distribution des dipôles électrostatiques ayant produit les potentiels électriques mesurés sur la peau par l'EEG au moyen des électrodes. De façon précise, nous cherchons à déterminer, le nombre de dipôles, leurs positions et leurs amplitudes.

Dans la suite de ce travail nous supposons les conductivités connues, et considérons un modèle couramment accepté de la tête, défini par une géométrie de couches concentriques représentant des milieux différents (Scalp, Crâne, Liquide Cérébrospinal, matières grise et blanche, etc...), chacun étant considéré comme homogène et isotrope (admettant une conductivité scalaire constante). Dans le cadre de ce travail, nous prenons un modèle de tête de 3 couches $\cup_{j=0}^{2} \Omega_{j}$ (Fig. 1), qui représentent de l'extérieur vers l'intérieur : $\Omega_{2}$ pour le Scalp, $\Omega_{1}$ pour le Crâne et $\Omega_{0}$ pour le Cerveau. Nous notons par $\Gamma_{j}$ l'interface entre $\Omega_{j}, \Omega_{j+1}$ pour $j=0,1$, par $\Gamma_{2}$ la surface extérieure du Scalp et par $S$ la partie de

TamTam'05 
$\Gamma_{2}$ où on connaît les mesures du potentiel obtenues par l'EEG.

Pour les résultats numériques, nous utiliserons particulièrement des modèles sphériques par simplicité. Le cas de modèles réalistes ne présente aucune difficulté supplémentaire.

Plusieurs approches ont été appliquées à la résolution du problème inverse en EEG. Parmi elles, celles qui consistent à minimiser une certaine fonctionnelle coût; ces méthodes calculent uniquement les positions et les moments des dipôles. Comme exemple de ces approches, nous citons la méthode de moindres carrées qui minimise l'erreur entre les données mesurées et celles calculées en résolvant un problème direct [9],[12]; et une autre méthode, dite de type Kohn et Vogelius, qui consiste à minimiser la différence énergétique entre deux problèmes directs écrits dans le domaine de la tête, l'un à condition de Dirichlet sur $\Gamma_{2}$ (formée à partir des mesures) et l'autre à condition de Neumann sur $\Gamma_{2}$ (formée par le flux de l'air qui est nul) ([7]). D'autres approches, récemment proposées, sont basées sur des méthodes analytiques comme l'approximation méromorphe ([2]) et les méthodes dites algébriques [8], [6], [15], [19], [22]. Ces méthodes ne sont applicables que dans des domaines homogènes, en l'occurence ici le cerveau $\left(\Omega_{0}\right)$ et requièrent la donnée du potentiel et de sa dérivée normale sur toute la surface du cerveau. Ce qui nécessite la résolution d'un, voire de plusieurs problèmes de Cauchy.

Le travail que nous présentons ici rentre dans le cadre de la résolution du problème inverse en EEG par la méthode proposée par A. El Badia \& T. Ha-Duong, [8],[6]. Celle ci est composée de deux parties. La première consiste à "remonter" les données du potentiel recueillies sur le scalp au cortex en résolvant un voire plusieurs problèmes de Cauchy. La deuxième partie consiste à déterminer la nombre de dipôles à l'intérieur du cerveau ainsi que leurs positions et leurs moments au moyen d'une méthode algébrique, sans procédure d'optimisation. La mise en oeuvre numérique de la deuxième partie est en cours de rédaction. Dans ce papier, nous nous intéressons uniquement à la résolution du problème de Cauchy.

Nous considérons donc le problème de détermination du potentiel et du flux sur la surface $\left(\Gamma_{0}\right)$ du cerveau à partir des données de Cauchy dont nous disposons à la surface du scalp $\left(\Gamma_{2}\right)$ en résolvant le problème suivant

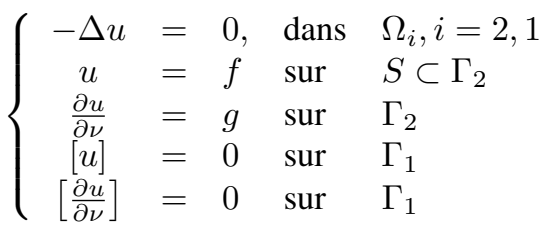




\section{TamTam'05}

avec $f$ et $g$ sont deux données compatibles avec $f \in H^{1 / 2}(S)$ et $g \in H^{-1 / 2}\left(\Gamma_{2}\right)$. Notons que dans le cas du problème inverse en EEG $g=0$ en raison de la non conductivité de l'air.

Pour la résolution numérique du problème (1) nous avons choisi une méthode alternative itérative initialement proposée par Kozlov, Maz’ya et Fomin [16]. Nous présontons deux approches, la première que nous nommons "approche successive" consiste à appliquer l'algorithme de Kozlov et al successivement, d'abord dans le scalp ensuite dans le crâne. La deuxième dite "approche simultanée" consiste à appliquer le même algorithme simultanément sur, le scalp et l'os.

La suite de cet article se compose de la façon suivante. Dans la deuxième section, nous poserons le problème de Cauchy et présonterons la méthode itérative de Kozlov, pour les deux approches "successive" et "simultanée". Nous donnerons également la formulation en équations intégrales des problèmes directs intervenant dans les deux approches. La troisième section est dédiée aux résultats numériques (implémentés sur un modèle sphérique) où nous comparerons entre les deux approches "successive" et "simultanée". Enfin, nous tirons quelques conclusions de ces expériences numériques à la dernière section.

\section{Résolution du problème de Cauchy}

Dans cette section, nous présentons les deux approches "successive" et "simultanée" pour résoudre notre problème de Cauchy. Mais avant cela, nous allons décrire brièvement ce problème et introduire la méthode choisie.

Le problème de Cauchy consiste à déterminer la solution sur les parties inaccessibles d'un domaine à partir des données dites "Données de Cauchy" connues partiellement sur la frontière. Ce problème admet une solution unique si les données sont compatibles (i.e. si elles constituent la trace et trace normale d'une certaine fonction harmonique). Mais, ce problème est mal-posé dans le sens où une légère perturbation des données à la frontière entraîne une grande incertitude sur la solution à l'intérieur du domaine [11].

Plusieurs méthodes de régularisation ont été proposées pour résoudre le problème de Cauchy. Nous citons parmi elles la méthode de quasi-réversibilité [17], [3], (voir aussi les référnces qui y sont citées) qui consiste à transformer le problème de Cauchy en un problème bien posé mais d'ordre plus élevé. Autres méthodes comme la méthode de régularisation de Tikhonov qui consiste à régulariser le problème de Cauchy à l'aide des

TamTam'05 
fonctions paramétriques [21], [5], (voir aussi les références qui y sont citées).

D'autre part, Kozlov \& al. ([16]) ont proposé une méthode alternative itérative régularisante pour résoudre le problème de Cauchy. Les paramètres de régularisation de cette méthode sont le nombre d'itérations et le choix initial de l'algorithme.

D'après [16], pour des données compatibles, nous obtenons une vraie convergence théorique mais pour des données bruitées il faudrait trouver un compromis entre le nombre d'itérations et la perturbation sur les mesures.

Nous avons choisi cette méthode pour résoudre notre problème de Cauchy. Elle est basée sur un procédé itératif alternatif qui consiste à résoudre une suite de problèmes elliptiques bien posés aux conditions limites mêlées. Nous référons à [16] pour l'étude théorique de cette méthode, dans le présent papier nous nous limitons à l'étude numérique appliquée à notre problème dans $R^{3}$. Notons par ailleurs qu'elle a été expérimentée dans d'autres applications $c f$. [13],[14],[18].

\subsection{Approche successive}

Cette approche consiste à résoudre le problème en deux étapes : On résoud d'abord le problème de Cauchy $\left(P C_{S c a l p}\right)$ dans le domaine formé par le scalp pour transmettre les données de $\Gamma_{2}$ à $\Gamma_{1}$ (Fig. 1); Ensuite résoudre un deuxième problème de Cauchy $\left(P C_{C r a ̂ n e}\right)$ dans le domaine formé par le crâne afin de déterminer le potentiel et le flux sur $\Gamma_{0}$. Ces deux problèmes s'écrivent comme suivant :

$$
\left(P C_{\text {Scalp }}\right) \begin{cases}-\Delta u_{2}=0 & \text { dans } \Omega_{2} \\ u_{2}=f & \text { sur } S \subset \Gamma_{2} \\ \frac{\partial u_{2}}{\partial \nu}=g & \text { sur } \Gamma_{2}\end{cases}
$$

La résolution du problème $\left(P C_{\text {Scalp }}\right)$ nous permet d'obtenir les traces du potentiel et du flux sur $\Gamma_{1}:\left(u_{2}\right)_{\mid \Gamma_{1}}$ et $\left(\partial_{\nu} u_{2}\right)_{\mid \Gamma_{1}}$.

$$
\left(P C_{\text {Crâne }}\right) \begin{cases}-\Delta u_{1}=0 & \text { dans } \Omega_{1} \\ u_{1}=u_{2} & \text { sur } \Gamma_{1} \\ \frac{\partial u_{1}}{\partial \nu}=-\frac{\sigma_{2}}{\sigma_{1}} \partial_{\nu} u_{2} & \text { sur } \Gamma_{1}\end{cases}
$$

La résolution du problème $\left(P C_{C r a ̂ n e}\right)$ nous permet d'obtenir les traces du potentiel et du flux sur $\Gamma_{0}$.

Ici $\sigma_{1}$ et $\sigma_{2}$ désignent respectivement la conductivité du scalp et celle de l'os et $\vec{\nu}$ le vecteur normal unitaire, qui est dirigé vers l'extérieur du domaine.

TamTam'05 


\section{TamTam'05}

Dans les deux cas, il s'agit de résoudre deux fois un problème de Cauchy dans une couronne qui s'écrit comme suivant :

$$
\left(P C_{\text {suc }}\right) \begin{cases}-\Delta u=0 & \text { dans } \Omega \\ u=f & \text { sur } S \subset \Gamma_{e} \\ \frac{\partial u}{\partial \nu}=g & \text { sur } \Gamma_{e}\end{cases}
$$

où $\Omega$ est une couronne dans $R^{3}$ (Fig. 2) représentant le domaine entre deux couches de la tête, $\Gamma_{e}$ la surface extérieure de la couronne et $\Gamma_{i}$ est la surface intérieure, $S$ une partie stricte de $\Gamma_{e}$ pour la première couronne (le Scalp), et $S=\Gamma_{e}$ pour la deuxième couronne (le Crâne) avec les données de Cauchy $f \in H^{1 / 2}(S)$ et $g \in H^{-1 / 2}\left(\Gamma_{e}\right)$ supposées compatibles. Notre but est de déterminer les valeurs inconnues du potentiel $u$ et du flux $\frac{\partial u}{\partial \nu} \operatorname{sur} \Gamma_{i}$.

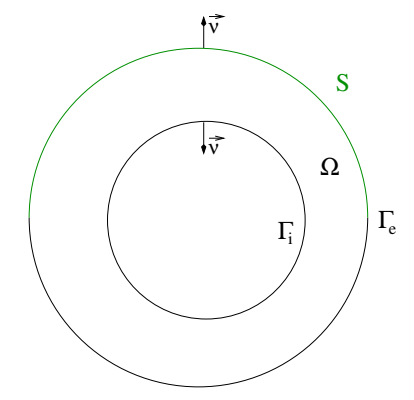

Figure 2.

\subsubsection{Le schéma de l'algorithme itératif de Kozlov dans une couronne}

L'algorithme proposé par Kozlov et al se base sur un choix initial quelconque d'une estimation du potentiel (ou du flux) sur la surface intérieure $\left(\Gamma_{i}\right)$. Et, il s'arrête quand les deux suites formées par la trace et la trace normale du potentiel sur $\Gamma_{i}$ deviennent stationnaire. Un autre critère d'arrêt pourrait être la stationnarité de la suite du potentiel ; ce choix sera justifié par les résultats numériques dans la section suivante.

Pour le problème $\left(P C_{\text {suc }}\right)$, l'algorithme est le suivant :

- Etant donné $f^{0} \in H^{1 / 2}\left(\Gamma_{i}\right)$ une approximation initiale du potentiel sur $\Gamma_{i}$.

- Résoudre :

$$
\left(P 1_{\text {suc }}\right) \begin{cases}-\Delta u^{2 n+1}=0 & \text { dans } \Omega \\ u^{2 n+1}=f^{n} \operatorname{sur} \Gamma_{i}, & \partial_{\nu} u^{2 n+1}=g\end{cases}
$$

TamTam'05 
pour obtenir $g^{n}=\left.\partial_{\nu} u^{2 n+1}\right|_{\Gamma_{i}}$

- Ensuite résoudre :

$\left(P 2_{\text {suc }}\right) \begin{cases}-\Delta u^{2 n+2}=0 & \text { dans } \Omega \\ \partial_{\nu} u^{2 n+2}=g^{n} \text { sur } \Gamma_{i}, & u^{2 n+2}=f \text { sur } S, \quad \partial_{\nu} u^{2 n+2}=g \text { sur } \Gamma_{e} \backslash S\end{cases}$

pour déterminer $f^{n+1}=u_{\left.\right|_{\Gamma_{i}}}^{2 n+2}$.

Ce schéma correspond au cas des données partielles. Dans le cas du passage Crâne/Cerveau, nous connaissons $u=f$ et $\frac{\partial u}{\partial \nu}=g$ sur toute $\Gamma_{e}$, et donc il faut changer convenablement les conditions aux limites dans le problème $\left(P 2_{\text {suc }}\right)$, il s'agit alors de remplacer les deux conditions sur $\Gamma_{e}$ par la condition de Dirichlet suivante $: u^{2 n+2}=f$ sur $\Gamma_{e}$.

\subsection{Approche simultanée}

L'approche simultanée consiste à appliquer l'algorithme de Kozlov et al directement au problème posé dans le domaine constitué du scalp et de l'os comme suit :

$$
\left(P C_{\text {sim }}\right)\left\{\begin{array}{lll}
-\Delta u & =0 & \text { dans } \Omega_{1} \cup \Omega_{2} \\
{[u]} & =0 & \text { sur } \Gamma_{1} \\
{\left[\sigma \partial_{\nu} u\right]} & =0 & \text { sur } \Gamma_{1} \\
u & =f & \text { sur } S \subset \Gamma_{2} \\
\frac{\partial u}{\partial \nu} & =g & \text { sur } \Gamma_{2}
\end{array}\right.
$$

où $[u]=u^{-}-u^{+}$avec $u^{-}$désigne la limite intérieure $\left(\lim _{\epsilon \rightarrow 0^{-}} u(x+\epsilon \vec{\nu})\right)$ et $u^{+}$la limite extérieure $\left(\lim _{\epsilon \rightarrow 0^{+}} u(x+\epsilon \vec{\nu})\right)$.

\subsubsection{Le schéma de l'algorithme simultanément dans deux couronnes}

Cet algorithme s'écrit comme suit :

- Etant donné $f^{0} \in H^{1 / 2}\left(\Gamma_{0}\right)$ une approximation initiale du potentiel sur $\Gamma_{0}$.

- Résoudre :

$$
\left(P 1_{\text {sim }}\right)\left\{\begin{array}{lll}
-\Delta u^{2 n+1}=0 & \text { dans } \Omega_{1} \cup \Omega_{2} & \\
{\left[u^{2 n+1}\right]=0 \text { sur } \Gamma_{1},} & {\left[\sigma \partial_{\nu} u^{2 n+1}\right]=0} & \text { sur } \Gamma_{1} \\
u^{2 n+1}=f^{n} \operatorname{sur} \Gamma_{0}, & \partial_{\nu} u^{2 n+1}=g & \text { sur } \Gamma_{2}
\end{array}\right.
$$

$\operatorname{Poser} g^{n}=\left.\partial_{\nu} u^{2 n+1}\right|_{\Gamma_{0}}$ 


\section{TamTam'05}

- Puis résoudre :

$$
\left(P 2_{\text {sim }}\right)\left\{\begin{array}{lll}
-\Delta u^{2 n+2}=0 & \text { dans } \Omega_{1} \cup \Omega_{2} \\
{\left[u^{2 n+2}\right]=0 \operatorname{sur} \Gamma_{1},} & {\left[\sigma \partial_{\nu} u^{2 n+2}\right]=0} & \text { sur } \Gamma_{1} \\
\partial_{\nu} u^{2 n+2}=g^{n} \operatorname{sur} \Gamma_{0}, & u^{2 n+2}=f \text { sur } S, & \partial_{\nu} u^{2 n+2}=g \text { sur } \Gamma_{2} \backslash S
\end{array}\right.
$$

Poser $f^{n+1}=u_{\left.\right|_{\Gamma_{0}}}^{2 n+2}$.

\subsection{La résolution d'un problème aux conditions limites mêlées}

A chaque itération de l'algorithme nous avons un problème du Laplacien aux conditions mêlées.

Pour l'approche successive nous avons à résoudre les problèmes suivants :

$$
\left(P 1_{\text {suc }}\right)\left\{\begin{array} { l l l } 
{ - \Delta u } & { = 0 } & { \text { dans } \Omega } \\
{ u } & { = \phi } & { \text { sur } \Gamma _ { i } } \\
{ \frac { \partial u } { \partial \nu } } & { = g } & { \text { sur } \Gamma _ { e } }
\end{array} \quad ( P 2 _ { \text { suc } } ) \left\{\begin{array}{lll}
-\Delta u=0 & \text { dans } \Omega \\
\partial_{\nu} u & =\psi & \text { sur } \Gamma_{i} \\
u & =f & \text { sur } S \subset \Gamma_{e} \\
\frac{\partial u}{\partial \nu} & =g & \text { sur } \Gamma_{e} \backslash S
\end{array}\right.\right.
$$

ici $\phi$ et $\psi$ représentent des fonctions appartenant respectivement à $H^{1 / 2}\left(\Gamma_{i}\right)$ et $H^{-1 / 2}\left(\Gamma_{i}\right)$, et $f$ et $g$ sont les données de Cauchy $\left(f \in H^{1 / 2}(S), g \in H^{-1 / 2}\left(\Gamma_{e}\right)\right)$. Le domaine $\Omega$ désigne la couronne mentionnée par (Fig 2).

Et, pour l'approche simultanée nous avons à résoudre :

$\left(P 1_{\text {sim }}\right)\left\{\begin{array}{lll}-\Delta u & =0 & \text { dans } \Omega_{1} \cup \Omega_{2} \\ u & =\phi & \text { sur } \Gamma_{0} \\ {[u]} & =0 & \text { sur } \Gamma_{1} \\ {\left[\sigma \frac{\partial u}{\partial \nu}\right]} & =0 & \text { sur } \Gamma_{1} \\ \frac{\partial u}{\partial \nu} & =g & \text { sur } \Gamma_{2}\end{array} \quad\left(P 2_{\text {sim }}\right)\left\{\begin{array}{lll}-\Delta u & =0 & \text { dans } \Omega_{1} \cup \Omega_{2} \\ \frac{\partial u}{\partial \nu} & =\psi & \text { sur } \Gamma_{0} \\ {[u]} & =0 & \text { sur } \Gamma_{1} \\ {\left[\sigma \partial_{\nu} u\right]} & =0 & \text { sur } \Gamma_{1} \\ u & =f & \text { sur } S \subset \Gamma_{2} \\ \frac{\partial u}{\partial \nu} & =g & \text { sur } \Gamma_{2} \backslash S\end{array}\right.\right.$

avec $\phi \in H^{1 / 2}\left(\Gamma_{0}\right)$ et $\psi \in H^{-1 / 2}\left(\Gamma_{0}\right), f \in H^{1 / 2}(S)$ et $g \in H^{-1 / 2}\left(\Gamma_{2}\right)$ (Fig 1$)$.

Ces problèmes sont bien posés pour des données compatibles. Pour les résoudre, nous utilisons la méthode des éléments de frontière (équations intégrales). D'une part, elle est bien adaptée à la géométrie des domaines considérés. D'autre part, comme nous n'avons pas besoin des valeurs intérieures aux domaines, nous avons préféré éviter la méthode d'éléments finis qui nous obligerait à travailler sur un maillage $3 D$ plutôt compliqué, ainsi que sur des interpolations géométriques aux surfaces séparant les domaines.

TamTam'05 
Nous présentons dans le paragraphe suivant la formulation intégrale utilisée ainsi que les systèmes matriciels obtenus pour la résolution des problèmes directs dans les deux approches successive et simultanée. Pour simplifier la présentation, on suppose $S=\Gamma_{e}$, ce qui n'enlève rien à la généralisation.

\subsubsection{Formulation en équations intégrales du problème direct à une couronne}

Pour résoudre les problèmes directs $\left(P 1_{\text {suc }}\right)$ et $\left(P 2_{\text {suc }}\right)$ nous utilisons la formulation intégrale suivante ([10],[20]) :

$$
\frac{1}{2} u(y)=\int_{\partial \Omega}\left[G(x, y) \frac{\partial u}{\partial \nu_{x}}(x)-\frac{\partial}{\partial \nu_{x}} G(x, y) u(x)\right] d \gamma(x) \quad \text { pour } y \in \partial \Omega
$$

où $G(x, y)=\frac{1}{4 \pi\|x-y\|}$ est la solution fondamentale du Laplacien dans $R^{3}$ satisfaisant $-\Delta G=\delta_{0}$. En écrivant cette équation pour $\Gamma_{i}$ et $\Gamma_{e}$ séparément nous obtenons un système de relations portant sur le potentiel et le flux sur les deux surfaces.

Matriciellement, le système obtenu s'écrit :

$$
\underbrace{\left[\begin{array}{ll}
S_{i, i} & -D_{i, e} \\
S_{e, i} & -\frac{I_{e}}{2}-D_{e, e}
\end{array}\right]}_{A_{1}}\left(\begin{array}{l}
\left(\partial_{\nu} u\right)_{i} \\
u_{e}
\end{array}\right)=\underbrace{\left[\begin{array}{ll}
\frac{I_{i}}{2}+D_{i, i} & -S_{i, e} \\
D_{e, i} & -S_{e, e}
\end{array}\right]}_{A_{2}}\left(\begin{array}{l}
u_{i} \\
\left(\partial_{\nu} u\right)_{e}
\end{array}\right)
$$

où $I_{a}$ est l'opérateur identité sur $\Gamma_{a}$ et $\left(S_{a, b} v\right)(y)=\int_{\Gamma_{b}} G(x, y) v(x) d \gamma(x), \quad y \in \Gamma_{a}$, le potentiel de simple couche, et $\left(D_{a, b} u\right)(y)=\int_{\Gamma_{b}} \partial_{\nu_{x}} G(x, y) u(x) d \gamma(x), \quad y \in \Gamma_{a}$ potentiel de double couche, $u_{e}=u_{\mid \Gamma_{e}}, u_{i}=u_{\mid \Gamma_{i}}\left(\partial_{\nu} u\right)_{i}=\left.\frac{\partial u}{\partial \nu}\right|_{\Gamma_{i}}$ et $\left(\partial_{\nu} u\right)_{e}=\frac{\partial u}{\partial \nu} \mid \Gamma_{e}$.

Une méthode de Galerkin, avec des fonctions de base $P^{1}$, a été choisie pour discrétiser ce système d'équations intégrales. Les intégrales faiblement singulières (qui apparaissent dans le système discrétisé) ont été calculées par une méthode semi-analytique ([10]). Et, les intégrales régulières ont été approchées par une méthode de quadrature de Gauss à 7 points.

Notons que dans le cas du problème aux conditions mêlées $\left(P 1_{s u c}\right)$ qui apparaît dans la suite impaire de l'algorithme de Kozlov, nous inversons la matrice $A_{1}$, et pour le problème $\left(P 2_{\text {suc }}\right)$ nous inversons la matrice $A_{2}$.

\subsubsection{Formulation en équations intégrales du problème direct à deux couronnes}

Pour résoudre les problèmes directs $\left(P 1_{\text {sim }}\right)$ et $\left(P 2_{\text {sim }}\right)$ nous utilisons la même formulation intégrale en simple et double couche (décrite au-dessus). Nous l'écrivons dans $\Omega_{1}$ 


\section{TamTam'05}

et dans $\Omega_{2}$, et nous prenons la trace sur leurs frontières respectives. Nous obtenons alors :

dans $\Omega_{2}$ :

$$
\left\{\begin{array}{lll}
\operatorname{sur} \Gamma_{2}: & -D_{2,1} u_{1}+S_{2,1}\left(\partial_{\nu} u\right)_{1}^{+} & =-\left(\frac{I_{2}}{2}+D_{2,2}\right) u_{2}+S_{2,2}\left(\partial_{\nu} u\right)_{2}^{-} \\
\operatorname{sur} \Gamma_{1}: & \left(\frac{I_{1}}{2}-D_{1,1}\right) u_{1}+S_{1,1}\left(\partial_{\nu} u\right)_{1}^{+} & =-D_{1,2} u_{2}+S_{1,2}\left(\partial_{\nu} u\right)_{2}^{-}
\end{array}\right.
$$

et dans $\Omega_{1}$ :

$$
\left\{\begin{array}{lll}
\operatorname{sur} \Gamma_{1}: & \left(\frac{I_{1}}{2}+D_{1,1}\right) u_{1}-S_{1,1}\left(\partial_{\nu} u\right)_{1}^{-} & =D_{1,0} u_{0}-S_{1,0}\left(\partial_{\nu} u\right)_{0}^{+} \\
\operatorname{sur} \Gamma_{0}: & D_{0,1} u_{1}-S_{0,1}\left(\partial_{\nu} u\right)_{1}^{-} & =\left(-\frac{I_{0}}{2}+D_{0,0}\right) u_{0}-S_{0,0}\left(\partial_{\nu} u\right)_{0}^{+}
\end{array}\right.
$$

Notons que ici le vecteur normal $\vec{\nu}$ est orienté vers l'extérieur comme le montre la figure (Fig. 1), et $\left(\partial_{\nu} u\right)_{j}^{-}$est la limite intérieure du flux à travers la surface $\Gamma_{j}$ et $\left(\partial_{\nu} u\right)_{j}^{+}$est la limite extérieure.

Nous considérons les deux équations écrites sur $\Gamma_{1}$, en tenant compte de la continuité de $\sigma \partial_{\nu} u$, nous exprimons $u_{1}$ et $\sigma_{1}\left(\partial_{\nu} u\right)_{1}^{-}$en fonction des valeurs sur $\Gamma_{0}$ et $\Gamma_{2}$. Puis, nous les remplaçons dans les équations écrites sur $\Gamma_{0}$ et $\Gamma_{2}$. Pour obtenir à la fin le système suivant :

$$
\begin{gathered}
{\left[\begin{array}{ll}
A_{00} & A_{02} \\
A_{20} & A_{22}
\end{array}\right]\left(\begin{array}{l}
\sigma_{0}\left(\partial_{\nu} u\right)_{0}^{-} \\
u_{2}
\end{array}\right)=\left[\begin{array}{ll}
B_{00} & B_{02} \\
B_{20} & B_{22}
\end{array}\right]\left(\begin{array}{l}
u_{0} \\
\sigma_{2}\left(\partial_{\nu} u\right)_{2}^{-}
\end{array}\right)} \\
\left\{\begin{array}{l}
A_{00}=S_{0,0}-\frac{\sigma_{1}}{\sigma_{1}-\sigma_{2}} D_{0,1} H_{1,1}^{-1} S_{1,0}-\frac{\sigma_{2}}{\sigma_{1}-\sigma_{2}} S_{0,1} S_{1,1}^{-1} H_{1,1}^{-1}\left(\frac{I_{1}}{2}-D_{1,1}\right) S_{1,0} \\
A_{02}=-\frac{\sigma_{1} \sigma_{2}}{\sigma_{1}-\sigma_{2}} D_{0,1} H_{1,1}^{-1} D_{1,2}+\frac{\sigma_{1} \sigma_{2}}{\sigma_{1}-\sigma_{2}} S_{0,1} S_{1,1}^{-1} H_{1,1}^{-1}\left(\frac{I_{1}}{2}+D_{1,1}\right) D_{1,2} \\
A_{20}=\frac{\sigma_{2}}{\sigma_{1}-\sigma_{2}} D_{2,1} H_{1,1}^{-1} S_{1,0}+\frac{\sigma_{2}}{\sigma_{1}-\sigma_{2}} S_{2,1} S_{1,1}^{-1} H_{1,1}^{-1}\left(\frac{I_{1}}{2}-D_{1,1}\right) S_{1,0} \\
A_{22}=\sigma_{2}\left(\frac{I_{2}}{2}+D_{2,2}\right)+\frac{\sigma_{2}{ }^{2}}{\sigma_{1}-\sigma_{2}} D_{2,1} H_{1,1}^{-1} D_{1,2}-\frac{\sigma_{1} \sigma_{2}}{\sigma_{1}-\sigma_{2}} S_{2,1} S_{1,1}^{-1} H_{1,1}^{-1}\left(\frac{I_{1}}{2}+D_{1,1}\right) D_{1,2} \\
\text { et } \begin{array}{l}
B_{00}=-\sigma_{1}\left(\frac{I_{0}}{2}-D_{0,0}\right)-\frac{\sigma_{1}{ }^{2}}{\sigma_{1}-\sigma_{2}} D_{0,1} H_{1,1}^{-1} D_{1,0}-\frac{\sigma_{1} \sigma_{2}}{\sigma_{1}-\sigma_{2}} S_{0,1} S_{1,1}^{-1} H_{1,1}^{-1}\left(\frac{I_{1}}{2}-D_{1,1}\right) D_{1,0} \\
B_{02}=-\frac{\sigma_{1}}{\sigma_{1}-\sigma_{2}} D_{0,1} H_{1,1}^{-1} S_{1,2}+\frac{\sigma_{1}}{\sigma_{1}-\sigma_{2}} S_{0,1} S_{1,1}^{-1} H_{1,1}^{-1}\left(\frac{I_{1}}{2}+D_{1,1}\right) S_{1,2} \\
B_{20}=\frac{\sigma_{1} \sigma_{2}}{\sigma_{1}-\sigma_{2}} D_{2,1} H_{1,1}^{-1} D_{1,0}+\frac{\sigma_{1} \sigma_{2}}{\sigma_{1}-\sigma_{2}} S_{2,1} S_{1,1}^{-1} H_{1,1}^{-1}\left(\frac{I_{1}}{2}-D_{1,1}\right) D_{1,0} \\
B_{22}=S_{2,2}+\frac{\sigma_{2}}{\sigma_{1}-\sigma_{2}} D_{2,1} H_{1,1}^{-1} S_{1,2}-\frac{\sigma_{1}}{\sigma_{1}-\sigma_{2}} S_{2,1} S_{1,1}^{-1} H_{1,1}^{-1}\left(\frac{I_{1}}{2}+D_{1,1}\right) S_{1,2}
\end{array}
\end{array}\right.
\end{gathered}
$$

TamTam'05 
où $\sigma_{j}$ est la conductivité de $\Omega_{j}(j=0,1,2)$ et $H_{1,1}=\left(\frac{\sigma_{1}+\sigma_{2}}{\sigma_{1}-\sigma_{2}}\right) \frac{I_{1}}{2}+D_{1,1}$.

\section{Résultats numériques}

Pour les applications numériques, nous avons utilisé trois surfaces sphériques concentriques (centrées à l'origine) représentant de l'extérieur vers l'intérieur : le Scalp, le Crâne et le Cerveau. Les sphères sont de rayons respectifs $1,0.92$ et 0.87 . Ces surfaces ont été

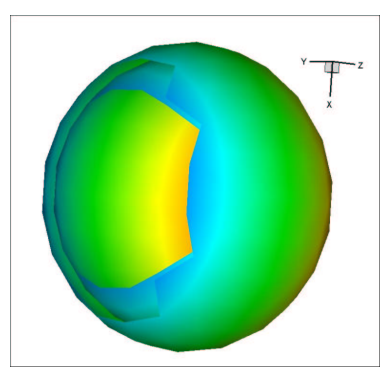

discrétisées par des facettes planes triangulaires formées de 320 triangles et 162 noeuds par couche.

Pour les conductivités, nous avons pris les valeurs utilisées dans la littérature et qui sont : 1 pour le Scalp et le Cerveau et 1/80 pour le Crâne.

Nous présentons dans un premier temps les résultats obtenus dans une couronne pour une fonction test en considérant le cas des données complètes et incomplètes. Ensuite nous donnons les résultats obtenus sur tout le domaine de la tête en partant des données calculées par nos soins afin de comparer entre les deux approches "successive" et "simultanée".

\subsection{Résultats numériques dans une couronne}

Nous considérons la couronne formée par les deux sphères de rayons respectifs 1 et 0.92 . Comme fonction harmonique, nous utilisons $u(x, y, z)=\frac{x}{r^{3}}=\frac{x}{\left(x^{2}+y^{2}+z^{2}\right)^{3 / 2}}$ dont nous obtenons explicitement les traces $u$ et $\frac{\partial u}{\partial \nu}$ sur $\Gamma_{e}$ et $\Gamma_{i}$. Nous déterminons ensuite les traces $u$ et $\frac{\partial u}{\partial \nu}$ sur $\Gamma_{i}$ en résolvant le problème de Cauchy en partant des données explicites sur $\Gamma_{e}$. Enfin nous comparons les résultats calculés aux valeurs exactes sur $\Gamma_{i}$. 


\section{TamTam'05}

\subsubsection{Cas de données complètes sur le bord extérieur}

Nous supposons les mesures connues sur toute la surface extérieure de la couronne et nous étudions le comportement de l'algorithme en fonction du choix initial $f^{0}$, en fonction de l'épaisseur de la couronne et en fonction de la perturbation des données.

\subsubsection{Résultats obtenus en fonction du choix initial}

Pour le choix de l'estimation initiale $f^{0}$ du potentiel $u_{i}$, nous avons pris $f^{0}=0$ et $f^{0}$ quelconque sur la surface intérieure $\Gamma_{i}$. Nous traçons dans la figure (Fig. 3) le logarithme en base 10 de l'erreur relative en norme $L^{2}$ sur $\Gamma_{i}$ entre les valeurs calculées du potentiel $u_{i}$ et du flux $\frac{\partial u}{\partial \nu}{ }_{i}$ et les valeurs exactes en fonction du nombre d'itérations.

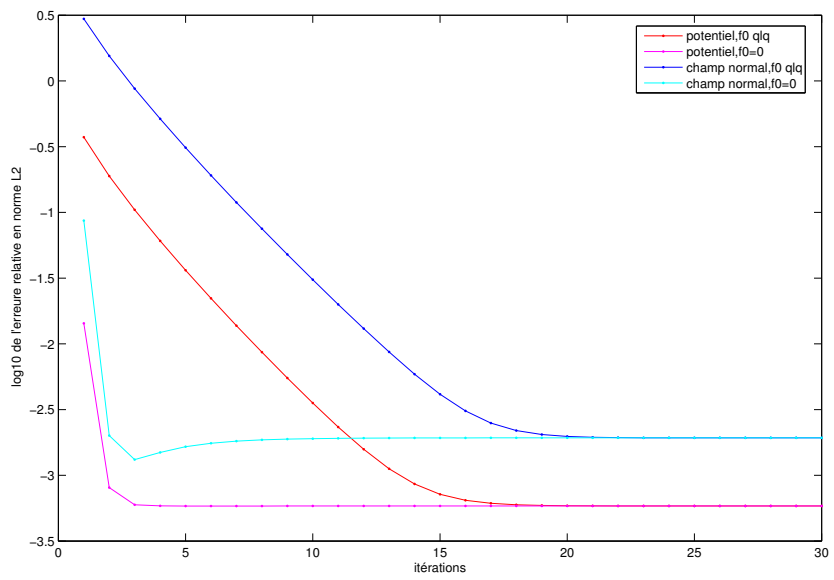

\section{Figure 3.}

Nous constatons alors que la suite calculée $\left(f^{n}\right)_{n}$ du potentiel sur $\Gamma_{i}$ tend vers une solution proche de la solution exacte avec une précision relative d'ordre $0.058 \%$ à partir de la 5ème itération pour un choix initial nul et à partir de la 16ème itération pour un choix initial quelconque.

Egalement, la suite du flux $\left(g^{n}\right)_{n}$ évolue vers une solution proche de la solution exacte avec une précision relative d'ordre $0.18 \%$ à partir de la 13ème itération pour un choix initial nul et à partir de la 20ème itération pour un choix initial quelconque.

Ces résultats nous permettent de constater que le choix initial n'influe pas sur la précision mais seulement sur le nombre d'itération, et que la précision relative sur le flux est

TamTam'05 
moins bonne que celle sur le potentiel ; résultat obtenu par D. Lesnic \& al. [18] dans leurs applications en $2 D$. Nous concluons également que les deux suites calculées deviennent stationnaires avec une bonne précision et ceci avec peu d'itérations ce qui pourrait être expliqué par la faible épaisseur de la couronne.

D'après ces résultats, nous constatons également que les suites calculées sur $\Gamma_{i}$ du potentiel et du flux deviennent stationnaires à peu d'itérations de différence, ce qui justifie le critère d'arrêt que nous avons choisi.

Dans ce qui suit, nous prenons $f^{0}=0$.

\subsubsection{Résultats obtenus en fonction de l'épaisseur}

Nous reprenons la même couronne, fixons le rayon de $\Gamma_{e}$ à 1 , et varions celui de $\Gamma_{i}$ en prenant les valeurs suivantes : $0.3,0.4,0.5,0.6,0.7,0.8,0.9,0.95,0.98$.

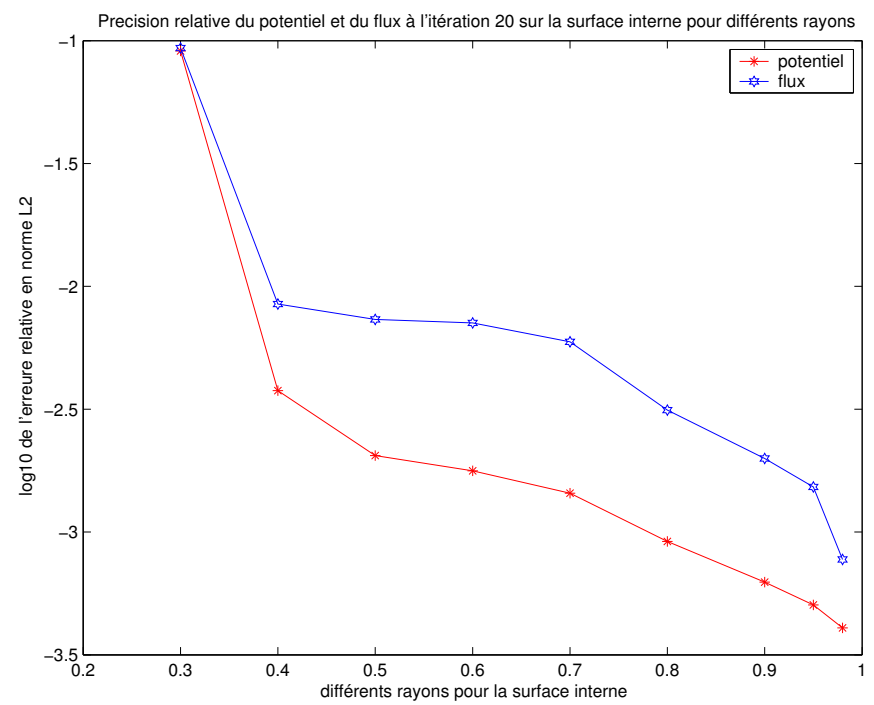

\section{Figure 4.}

Dans la figure (Fig. 4) nous traçons l'erreur relative en norme $L^{2}$ pour le potentiel et le flux calculés sur la surface interne et ceci pour les différentes valeurs de son rayon (valeurs prises à la 20ème itération de l'algorithme). 


\section{TamTam'05}

Nous remarquons alors, de façon relativement intuitive, que la précision sur le calcul du potentiel et du flux augmente avec la diminution de l'épaisseur de la couronne. Ce qui "valide" notre choix de cette méthode pour résoudre le problème de Cauchy en EEG.

\subsubsection{Etude du comportement de l'algorithme en fonction de la perturbation des données}

Dans ce paragraphe nous étudions le comportement de l'algorithme de Kozlov et al en fonction des perturbations des données sur la surface extérieure de la couronne. Nous ajoutons aux données $u_{e}$ et $\left(\partial_{\nu} u\right)_{e}$ un bruit gaussien d'amplitudes respectives $p=0,0.1$, $0.5,1,3$, et $5 \%$.

Nous traçons dans les figures (Fig. 5) et (Fig. 6) respectivement la variation de l'erreur relative sur le potentiel et le flux calculés sur $\Gamma_{i}$ en fonction du nombre d'itérations pour des données perturbées.

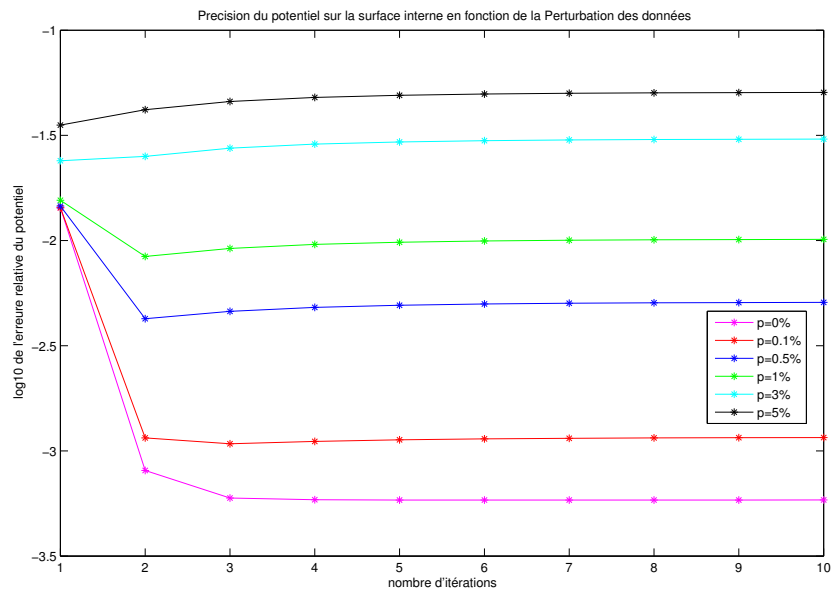

Figure 5.

Nous remarquons sur ces deux figures que l'algorithme de Kozlov et al est sensible aux perturbations des données sur le bord extérieur de la couronne, et surtout le calcul du flux qui devient de plus en plus imprécis avec l'augmentation de la perturbation.

Ces résultats nous montrent également l'aspect régularisant de l'algorithme pour de faibles perturbations. Mais pour de grandes perturbations, cet algorithme tel quel n'est plus effi-

TamTam'05 


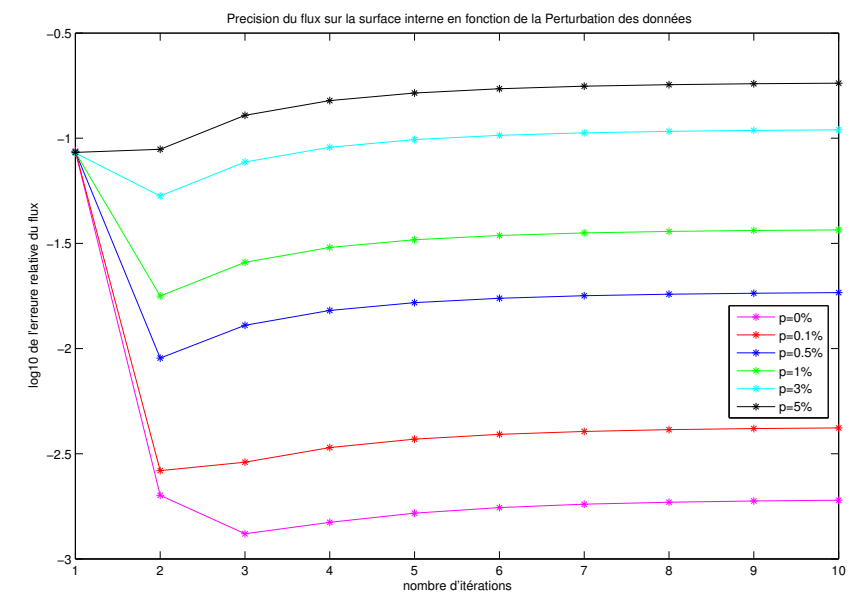

Figure 6.
cace.

Cependant, dans le cadre de notre problème EEG, ce qui nous intéresse est surtout de pouvoir calculer les Reciprocity Gap dans le domaine du Cerveau à partir des mesures. Pour cela, nous avons été amenés à trouver une autre alternative pour calculer les Reciprocity Gap à la surface du cerveau sans essayer de transférer les données bruitées du scalp jusqu'à la surface du cerveau, et ceci en essayant de ramener le calcul des Reciprocity Gap directement aux valeurs connues au Scalp, ce qui revient à résoudre un problème de Cauchy sur des fonctions tests en partant du Cerveau jusqu'au Scalp. Ce travail est en cours de rédaction.

\subsubsection{Cas de données incomplètes sur le bord extérieur}

Nous rappelons que dans ce cas, nous connaissons le potentiel partiellement sur la frontière extérieure : $u=f$ sur $S \subset \Gamma_{e}$.

Pour définir numériquement la partie $S$, nous définissons la variable $\alpha \in[-1 ; 1]$ telle que $S$ soit formée par les noeuds $X_{i}$ du maillage qui satisfont $X_{i}(3) \geq \alpha$. La figure (Fig. 7) représente $S$ pour quatre valeurs différentes de $\alpha$ qui sont (de gauche à droite et de haut en bas) : $-1,-0.8,-0.4$ et 0 .

La figure (Fig. 8) montre la variation de l'erreur relative sur le potentiel et le flux calculés $\left.\left(u_{i}, \partial_{\nu} u\right)_{i}\right)$ en fonction de différentes valeurs de $\alpha$ allant de $-1\left(S=\Gamma_{e}\right)$ jusqu'à $0.8(S$ représente $10 \%$ de $\Gamma_{e}$ ). 
TamTam'05

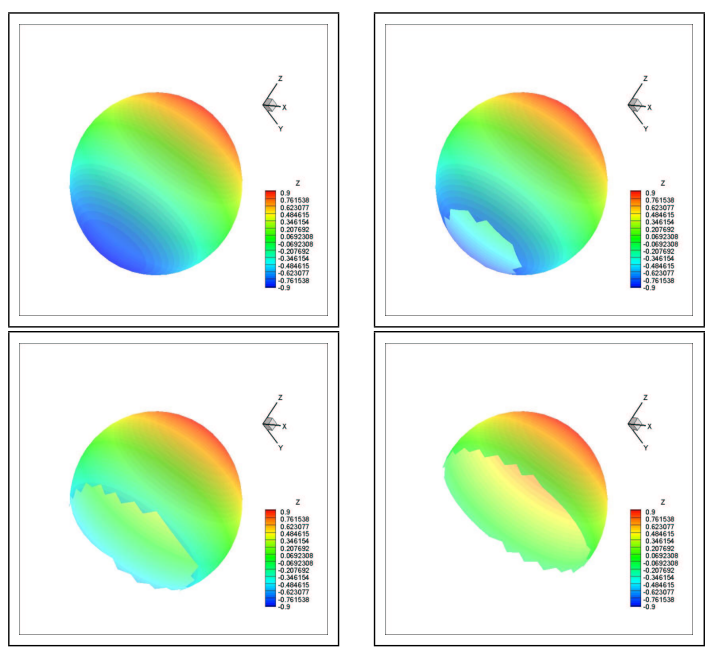

Figure 7.

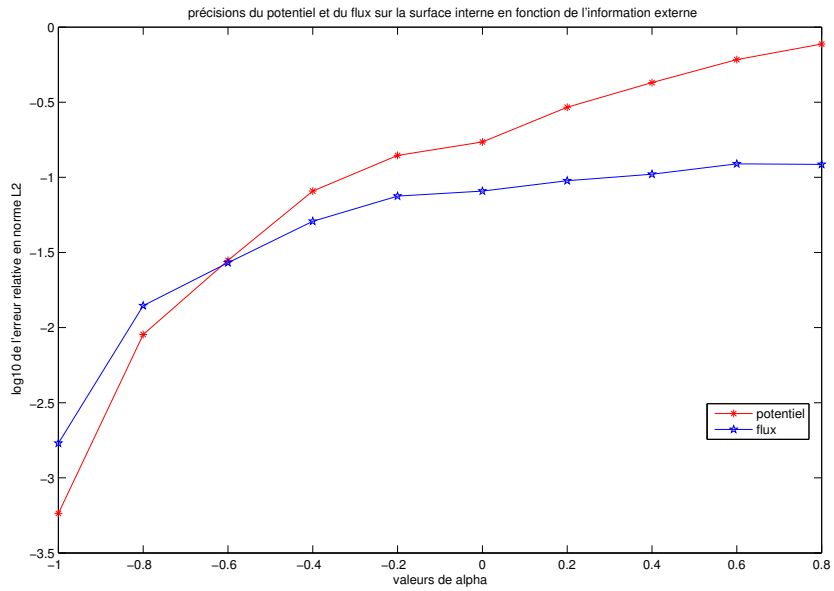

Figure 8.

TamTam'05 
Nous observons que l'erreur sur les valeurs calculées sur la surface intérieure augmente lorsque la surface des mesures diminue.

\subsection{Résultats numériques dans deux couronnes}

Nous présentons dans cette section les résultats obtenus en calculant les valeurs inconnues sur la surface du cerveau $\Gamma_{0}$ à partir des données de Cauchy sur la surface du scalp $\Gamma_{2}$, et ceci en faisant tourner l'algorithme d'abord successivement sur les deux couronnes et puis simultanément.

Pour cela, nous avons utilisé des données réelles calculées par nos soins, en résolvant un problème direct pour un dipôle placé dans la région profonde du cerveau. Nous avons considéré que $S$ représente $70 \%$ et $80 \%$ de la surface du scalp, et nous avons ajouté aux mesures un bruit de $3 \%$ et $5 \%$.

Nous donnons dans le tableau ci-contre l'erreur relative en norme $L^{2}$ sur les valeurs calculées sur le cerveau :

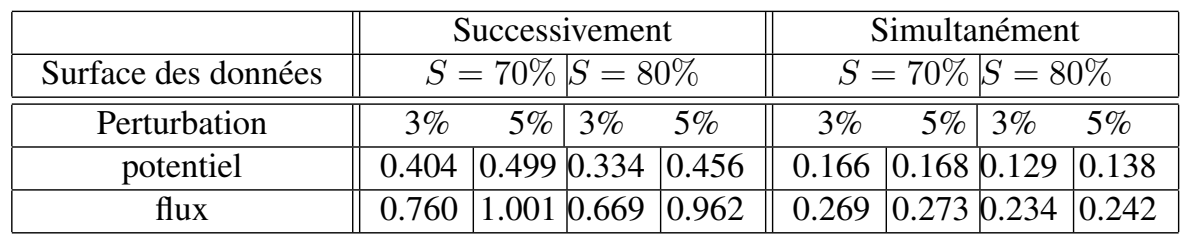

D'après ce tableau, il est clair que l'approche simultanée donne de meilleurs résultats que l'approche successive. Ceci pourrait être expliqué par le fait que dans l'approche successive, pour passer de la première couronne à la deuxième (voir $\left(P C_{C r a ̂ n e}\right)$ dans la section 2.1) nous multiplions la donnée du flux par $\frac{\sigma_{2}}{\sigma_{1}}:\left(\partial_{\nu} u_{1}\right)_{\mid \Gamma_{1}}=-\frac{\sigma_{2}}{\sigma_{1}}\left(\partial_{\nu} u_{2}\right)_{\mid \Gamma_{1}}$. Or ce facteur de conductivités vaut 80 , et la valeur $\left(\partial_{\nu} u_{2}\right)_{\mid \Gamma_{1}}$ est calculée à partir d'un problème de Cauchy, donc elle s'écrit : $\left(\partial_{\nu} u_{2}\right)_{\mid \Gamma_{1}}=\left(\partial_{\nu} u_{2}\right)_{\mid \Gamma_{1}}^{e x}+\epsilon$ où $\epsilon$ est l'erreur commise par rapport à la valeur exacte $\left(\partial_{\nu} u_{2}\right)_{\mid \Gamma_{1}}^{e x}$.

Donc, nous avons : $\left(\partial_{\nu} u_{1}\right)_{\mid \Gamma_{1}}=-80 \epsilon-80\left(\partial_{\nu} u_{2}\right)_{\mid \Gamma_{1}}^{e x}$, ce qui amplifie clairement l'erreur commise.

\section{Conclusion et Perspectives}

L'implémentation de l'algorithme de Kozlov et al pour deux approches différentes dans le cadre du problème EEG ainsi que les tests réalisés sur des couronnes sphériques nous ont permis d'étudier son comportement numérique en $3 D$ et d'en tirer plusieurs conclusions 


\section{TamTam'05}

intéressantes.

D'une part, les applications numériques faites sur une couronne sphérique de faible épaisseur ont montré un bon comportement numérique assuré par un faible nombre d'itérations avec une bonne précision relative.

Cette précision diminue avec le manque d'informations sur la surface extérieure de la couronne et avec la perturbation des données.

D'autre part, la résolution du problème de Cauchy dans tout le domaine de la tête (modèle sphérique) nous a permis de conclure que résoudre le problème simultanément est beaucoup mieux de le résoudre successivement.

Pour pallier aux insuffisances de l'algorithme de Kozlov, dans un premier temps nous élaborerons une méthode de complétion de données. Ensuite nous tâcherons de transporter le calcul des Reciprocity Gap pour la méthode algébrique appliquée dans le cerveau, à un calcul sur la surface du scalp en fonction des mesures et des traces des fonctions tests sur le scalp. Ainsi, on serait amené à résoudre le problème de Cauchy en partant des données (traces des fonctions tests) sur la surface du cerveau pour les transmettre à la surface du scalp. L'intérêt de cette approche est qu'on résoudra un problème de Cauchy sur des données complètes et non bruitées. Ce travail est en cours de rédaction.

\section{Bibliographie}

[1] S. Andrieux , T.N. Baranger , A. Ben AbDa Solving Cauchy problems by minimizing an energy-like functional, Inverse Problems 22 (115-133), 2006.

[2] L. Baratchart , A. Ben Abda, F. Ben Hassen And J. Leblond, Recovery of pointwise sources or small inclusions in $2 \mathrm{D}$ domains and rational approximaion, Inverse Problems 21 (51-74), 2005.

[3] L. Bourgeols A mixed formulation of quasi-reversibility to solve the Cauchy problem for Laplace's equation, Inverse Problems 21 (1087-1104), 2005.

[4] M. ChAFIK , A. El-BAdia, T. HA-DuOnG, On some inverse EEG problems, Inverse Problems Eng. Mech. II (537-544), 2000.

[5] A. Cimetière , F. Delvare , M. Jaoua , F. Pons Solution of the Cauchy problem using iterated Tikhonov regularization, Inverse Problems 17 (553-570), 2001.

[6] A. EL-BADIA Inverse source problem in an anisotropic medium by boundary measurements, Inverse Problems 21 (1787-1806), 2005.

[7] A. El-BADiA, M. FARAH Identification of dipole sources in an elliptic equation from boundary measurements, à paraître dans J. of Inverse and Ill-posed Problems.

TamTam'05 
[8] A. El-Badia , T. HA-Duong, An inverse source problem in potential analysis, Inverse Problems 16 (651-663), 2000.

[9] O. Faugeras , F. Clément , R. Deriche , R. Kerivien, T. PAPadoupolo , J. Roberts , T. Viéville , F. Devernay , J. Gomes, G. Hermosillo , P. Kornprobst , D. LinGRAND The inverse EEG and MEG problems : The adjoint space approch I : The continuous case, INRIA research report 3673, 1999.

[10] J. Giroire, Mise en oeuvre de méthodes d'éléments finis de frontière, Cours de DEA, 1997.

[11] J. HAdAmard Lectures on Cauchy's Problem in Linear Partial Differential Equation, New York : Dover, 1953.

[12] M. HÄmÄläinen , R. HaRi , R. J. Ilmoniemi , J. KnuUtila , O. V. LounasmaA, Magnetoencephalographiy-theory, instrumentation, and application to noninvasive studies of working humain brain, Rev. Mod. Phys. 65 (413-497), 1993.

[13] M. Jourhmane, A. NACHAOUI, An alternating method for an inverse Cauchy problem, Numerical Algorithms 21 (247-260), 1999.

[14] M. Jourhmane, D. LeSniC, N.S. Mera, Relaxation procedures for an iterative algorithm for solving the Cauchy problem for the Laplace equation, Engineering Analysis with Boundary Elements 28 (655-665), 2004.

[15] H. KANG , H. LEE Identification of simple poles via boundary measurements and an application of EIT, Inverse Problems 20 (1853-1863), 2004.

[16] V.A. Kozlov , V.G. MaZ'Ya , A.V. Fomin, An iterative method for solving the cauchy problem for elliptic equation, Comput. Math. Phys. 31 (45-52), 1991.

[17] R. Lattès, J.L. Lions Méthode de Quasi-réversibilité et Applications, Paris : Dunod, 1967.

[18] D. Lesnic, L. Elliott, D.B. Ingham, « An iterative boundary element method for solving numerically the Cauchy problem for the Laplace equation », Engineering Analysis with Boundary Elements 20 (123-133), 1997.

[19] T. NARA , S. ANDO, «A projective method for an inverse source problem of the Poisson equation », Inverse Problems 19 (355-369), 2003.

[20] J.C. NÉDÉLEC Acoustic and Electromagnetic Equations : Integral Representations for Harmonic Problems, Springer, 2000.

[21] A.N. Tikhonov , V.Y. Arsenin Solutions of Ill-posed Problems, Washington: Winston and Sons, 1977.

[22] K. YAMATANi , K. OHNAKa A reliable estimation method of a dipole for three dimensional Poisson equation, Jour. of Comp. and Appl. Math. 95 (139-151), 1998. 